\title{
Specific DNA identification of Pheretima in the Naoxintong capsule
}

\author{
Xiaoxiao Zhu' ${ }^{1}$, Hoi-Yan Wu ${ }^{2}$, Pang-Chui Shaw ${ }^{2,3,4^{*}}$, Wei Peng ${ }^{1}$ and Weiwei Su ${ }^{1 *}$
}

\begin{abstract}
Background: Pheretima is a minister drug in Naoxintong capsule (NXTC), a well-known traditional Chinese medicine (TCM) formula for the treatment of cardiovascular and cerebrovascular diseases. Owing to the loss of morphological and microscopic characteristics and the lack of recognized chemical marker, it is difficult to identify Pheretima in NXTC. This study aims to evaluate the feasibility of using DNA techniques to authenticate Pheretima, especially when it is processed into NXTC.

Methods: DNA was extracted from crude drugs of the genuine and adulterant species, as well as nine batches of NXTCs. Based on mitochondrial cytochrome c oxidase subunit I (COI) gene, specific primers were designed for two genera of genuine species, Metaphire and Amynthas, respectively. PCR amplification was performed with the designed primers on crude drugs of Pheretima and NXTCs. The purified PCR products were sequenced and the obtained sequences were identified to species level with top hit of similarity with BLAST against GenBank nucleotide database.
\end{abstract}

Results: Primers MF2R2 and AF3R1 could amplify specific DNA fragments with sizes around 230-250 bp, both in crude drugs and NXTC. With sequencing and the BLAST search, identities of the tested samples were found.

Conclusion: This study indicated that the molecular approach is effective for identifying Pheretima in NXTC. Therefore, DNA identification may contribute to the quality control and assurance of NXTC.

Keywords: Pheretima, Naoxintong capsule, DNA identification, COI gene

\section{Background}

Naoxintong capsule (NXTC), a traditional Chinese medicine (TCM) for the prevention and treatment of cardiovascular and cerebrovascular diseases, is approved by the China Food and Drug Administration (CFDA) and recorded in the 2015 edition of Chinese Pharmacopoeia (ChP 2015) [1]. NXTC is developed from Bu-Yang-HuanWu-Tang, a traditional prescription which has been found effective clinically in the treatment of stroke and cerebral infarction since Qing dynasty [2]. Nowadays, several comprehensive and systematic studies have confirmed the clinical efficacy of NXTC for the treatment

\footnotetext{
*Correspondence: pcshaw@cuhk.edu.hk; Isssww@126.com

1 Guangdong Engineering and Technology Research Center for Quality and Efficacy Reevaluation of Post-Market Traditional Chinese Medicine, Guangdong Key Laboratory of Plant Resources, School of Life Sciences, Sun Yat-Sen University, Guangzhou 510275, China

${ }^{2}$ Li Dak Sum Yip Yio Chin R \& D Centre for Chinese Medicine, The Chinese

University of Hong Kong, Shatin, N.T., Hong Kong, China

Full list of author information is available at the end of the article
}

of patients with angina pectoris [3], cerebral infarction [4], transient ischemic attack [5], vertebra-basilar insufficiency [6] and carotid atherosclerosis $[7,8]$.

Each NXTC weighs $0.4 \mathrm{~g}$ and is composed of sixteen powdered TCMs, including thirteen plant drugs and three animal drugs, namely Pheretima, Hirudo and Scorpio. These three animal drugs account for only $16.7 \%$ $(\mathrm{w} / \mathrm{w})$ of all sixteen TCMs, but they are the major effective components of NXTC. They play vital roles in clearing and activating the meridians and collaterals [2]. Pheretima was documented as a TCM in the earliest known treatise of Chinese material medica, "Shennong Bencao Jing", written 2000 years ago [9], and was widely used for the treatment of fever, asthma and stroke [1]. Increasing evidences indicate that Pheretima has wide therapeutic properties, including anti-inflammatory activity [10], anti-oxidative activity [11], fibrinolytic activity [12], anti-asthmatic activity [13], as well as promoting tumor apoptosis [14] and bone regeneration [15]. 
According to ChP 2015, there are four Pheretima animals, namely Amynthas aspergillus E. Perrier, Amynthas pectiniferus Michaelsen, Metaphire vulgaris Chen and Metaphire guillelmi Michaelsen. However, according to a survey on the resources of Pheretima and examination of the available drug [16], there are thirteen original animals of Pheretima in the market, including some common adulterants not recorded in ChP 2015. In consideration of the efficacy and safety of Pheretima, it is necessary to authenticate the species accurately. Unlike plant drugs, animal drugs lack the representative markers or chemical profiles. In ChP 2015, morphological and microscopic observation and thin layer chromatography (TLC) are used to identify Pheretima. However, the above conventional methods lack specificity and depend on experience of the examiner. What is more, the morphological and microscopic characteristics of the constituting species are lost in NXTC due to the processing procedure. Thus, it is difficult to find whether the NXTC contains the genuine species of Pheretima.

DNA is unique to every individual and exists in most biological tissues. In recent years, DNA techniques have been widely applied in the identification of TCM $[17,18]$, and it has also been used to identify Pheretima [19-21]. In addition, DNA methods have been established to authenticate two medicinal snakes (Zaocys and Agkistrodon) and one Chinese herb (Fritillariae cirrhosae bulbus) in ChP 2015. Nevertheless, previous studies mainly focused on single TCM rather than complex prescriptions. In this study, we explored the possibility of using DNA techniques to identify Pheretima in NXTC.

\section{Methods \\ Sample collection}

Pheretima medicinal materials were collected from various pharmacies in China or provided by Shaanxi Buchang Pharmaceuticals Co., Ltd. (Shaanxi, China) (Table 1). All of them were authenticated based on their morphological features by Guangdong Institute for Drug Control (Guangdong, China) according to ChP 2015 and confirmed by DNA sequencing using the universal primers of mitochondrial cytochrome $c$ oxidase subunit I (COI) [22] (primer sequences are shown in Table 2). These authenticated Pheretima samples were deposited in School of Life Sciences, Sun Yat-Sen University. Nine batches of NXTC were provided by Shaanxi Buchang Pharmaceuticals Co., Ltd. (Shaanxi, China). In-house NXTCs were also prepared as controls according to the standardized procedure for the preparation of NXTC in ChP 2015. A total of seven batches of homemade NXTC using different identified genuine or adulterant Pheretima samples and one batch of Pheretima-deficient NXTC containing all listed TCMs except for Pheretima were made. Detailed

Table 1 Information of the studied samples

\begin{tabular}{|c|c|c|c|c|}
\hline Material & Description & Batch no. & Source & Sample identity \\
\hline \multirow[t]{9}{*}{ Pheretima } & Genuine & P1-P3 & Buchang & Metaphire vulgaris \\
\hline & & P4 & Buchang & Metaphire guillelmi \\
\hline & & P5 & Market & Metaphire vulgaris \\
\hline & & P6-P10 & Market & Amynthas aspergillus \\
\hline & Adulterant & P11 & Market & Metaphire magna ssp. \\
\hline & & $\mathrm{P} 12$ & Market & $\mathrm{N} / \mathrm{A}$ \\
\hline & & P13 & Market & $\mathrm{N} / \mathrm{A}$ \\
\hline & & P14 & Market & $\mathrm{N} / \mathrm{A}$ \\
\hline & & P15 & Market & Metaphire californica \\
\hline \multirow[t]{8}{*}{ NXT capsules } & & N1-N9 & Buchang & Pheretima: Metaphire vulgaris \\
\hline & & N10 & Homemade & Pheretima: P6 \\
\hline & & N11 & Homemade & Pheretima: P4 \\
\hline & & N13 & Homemade & Pheretima: P11 \\
\hline & & $\mathrm{N} 14$ & Homemade & Pheretima: P12 \\
\hline & & N15 & Homemade & Pheretima: P13 \\
\hline & & N16 & Homemade & Pheretima: P14 \\
\hline & & N17 & Homemade & Pheretima: P15 \\
\hline Pheretima-deficient NXTC & & T4840 & Homemade & \\
\hline
\end{tabular}

* All the crude drugs were identified via their morphological features by Guangdong Institute for Drug Control (Guangdong, China) according to ChP 2015 and confirmed by DNA sequencing using the universal primers of COI. Buchang: Shaanxi Buchang Pharmaceuticals Co., Ltd. (Shaanxi, China)

N/A not available 
Table 2 Primers for PCR amplification and sequencing

\begin{tabular}{|c|c|c|c|}
\hline Primer name & Sequence $\left(5^{\prime} \rightarrow 3^{\prime}\right)$ & Amplicon size (bp) & $\begin{array}{l}\text { Annealing } \\
\text { temperature } \\
\left({ }^{\circ} \mathrm{C}\right)\end{array}$ \\
\hline \multicolumn{4}{|c|}{ Universal primers of COI [22] } \\
\hline LCO1490 & GGTCAACAAATCATAAAGATATTGG & 650 & 40 \\
\hline HCO2198 & TAAACTTTCAGGGTGACCAAAAAATCA & & \\
\hline \multicolumn{4}{|l|}{ MF2R2 } \\
\hline COI Metaphire F2 & TTAGTGTCGTCCGCCGCAGTT & 232 & 58 \\
\hline COI Metaphire R2 & CTACTGCCCACACAAATAGTGGG & & \\
\hline \multicolumn{4}{|l|}{ AF3R1 } \\
\hline COIAAF3 & TTTGGAAACTGACTGCTCCCA & 247 & 55 \\
\hline COI AA R1 & CTAAAATTGATGAGGCACCC & & \\
\hline
\end{tabular}

information of the samples and materials are shown in Table 1.

\section{DNA extraction}

DNA was extracted from crude drugs with Rapid Genomic DNA kit (Biomed, Beijing, China). Broad-spectrum plant Rapid Genomic DNA kit (Biomed, Beijing, China) was used for NXTC according to manufacturers' instructions with brief modification, respectively. The kit for plant DNA extraction was chosen as $83.6 \%$ (w/w) of NXTC is made up of plant materials. Twenty microliter proteinase $\mathrm{K}$ (20 mg/ml, Biomed, China) was added at the first step to digest the animal drugs. The DNA of Pheretima-deficient NXTC and seven batches of homemade NXTC were extracted by the same means as NXTC. Finally, $100 \mu \mathrm{l}$ DNA solution was obtained and the concentration was measured by NanoDrop 2000C spectrophotometer (Thermo Fisher Scientific, US). DNA was then adjusted to $50.00 \mathrm{ng} / \mu \mathrm{l}$.

\section{Primer design}

DNA sequences of COI gene for species belonging to Metaphire genus and Amynthas genus (Additional file 1: Tables S1-1 to S1-2) were obtained from GenBank of National Center for Biotechnology Information (NCBI) and aligned using BioEdit 7 software [23]. Owing to the significant difference of DNA sequences between Metaphire genus and Amynthas genus, different primers were designed respectively, according to the polymorphic sites between the genuine and adulterant species. All the primers were designed using Primer 3 (http://primer3.ut. ee/) and the information is shown in Table 2.

\section{PCR amplification and DNA sequencing}

PCR was performed in a $15 \mu$ l reaction mixture containing $1.5 \mu \mathrm{l} \mathrm{10 \times} \mathrm{PCR} \mathrm{buffer} \mathrm{(200} \mathrm{mM} \mathrm{Tris-HCl,} \mathrm{pH} \mathrm{8.4,}$ $\left.200 \mathrm{mM} \mathrm{KCl}, 100 \mathrm{mM}(\mathrm{NH} 4)_{2} \mathrm{SO}_{4}\right), 1.5 \mu \mathrm{l} 25 \mathrm{mM} \mathrm{MgCl}_{2}$,
$1.2 \mu \mathrm{l} 2.5 \mathrm{mM}$ dNTP mixture, $0.75 \mu \mathrm{l} 10 \mu \mathrm{M}$ each of the forward and reverse primer, $0.5 \mu \mathrm{l}$ DNA sample and $0.1 \mu \mathrm{l}$ $5 \mathrm{U} / \mu \mathrm{l} \mathrm{Taq}$ polymerase. All the buffers and chemicals mentioned above were bought from Beijing Biomed Co., Ltd (Beijing, China). Reactions were conducted using Veriti 96-well Thermal Cycle (Applied Biosystems, Singapore) through initial denaturation at $95^{\circ} \mathrm{C}$ for $3 \mathrm{~min}$, then 39 cycles of denaturation at $95^{\circ} \mathrm{C}$ for $45 \mathrm{~s}$, annealing at indicated temperature for $45 \mathrm{~s}$ and extension at $72{ }^{\circ} \mathrm{C}$ for $1 \mathrm{~min}$, with a final extension at $72{ }^{\circ} \mathrm{C}$ for $5 \mathrm{~min}$. The PCR products were electrophoresed and visualized on 1.5\% agarose gels stained with SYBR Safe DNA gel stain (Thermo Fisher Scientific, US), purified with DNA gel purification kit (Biomed, Beijing, China) and sequenced by Sanger sequencing (Tech Dragon, Hong Kong and Beijing Genomics Institute, Guangzhou, China). By means of Basic Local Alignment Search Tool (BLAST) of NCBI, the amplified sequences were compared with public DNA sequences in GenBank nucleotide database to match the most likely species with the top hit.

\section{Results}

Identification of the animal drug by species-specific PCR

To verify the specificity of the designed primers, crude drugs for both the genuine and the adulterant were firstly tested. The common adulterants of Pheretima we collected on the market include $M$. magna ssp. and $M$. californica (Kinberg), as well as another three unidentifiable species. As shown in Fig. 1a, by using the specific primers MF2R2 for Metaphire genus, 232-bp fragments were amplified in the samples from P1 to P5. At the same time, no amplification was obtained from another genuine species in the Amynthas genus (P6-P10) and the adulterants (P11-P15). For AF3R1(Fig. 1b), the specific primers for Amynthas genus, 247-bp amplicons with sequences the same as the genuine species $(A$. aspergillus) were obtained, while no amplification was 


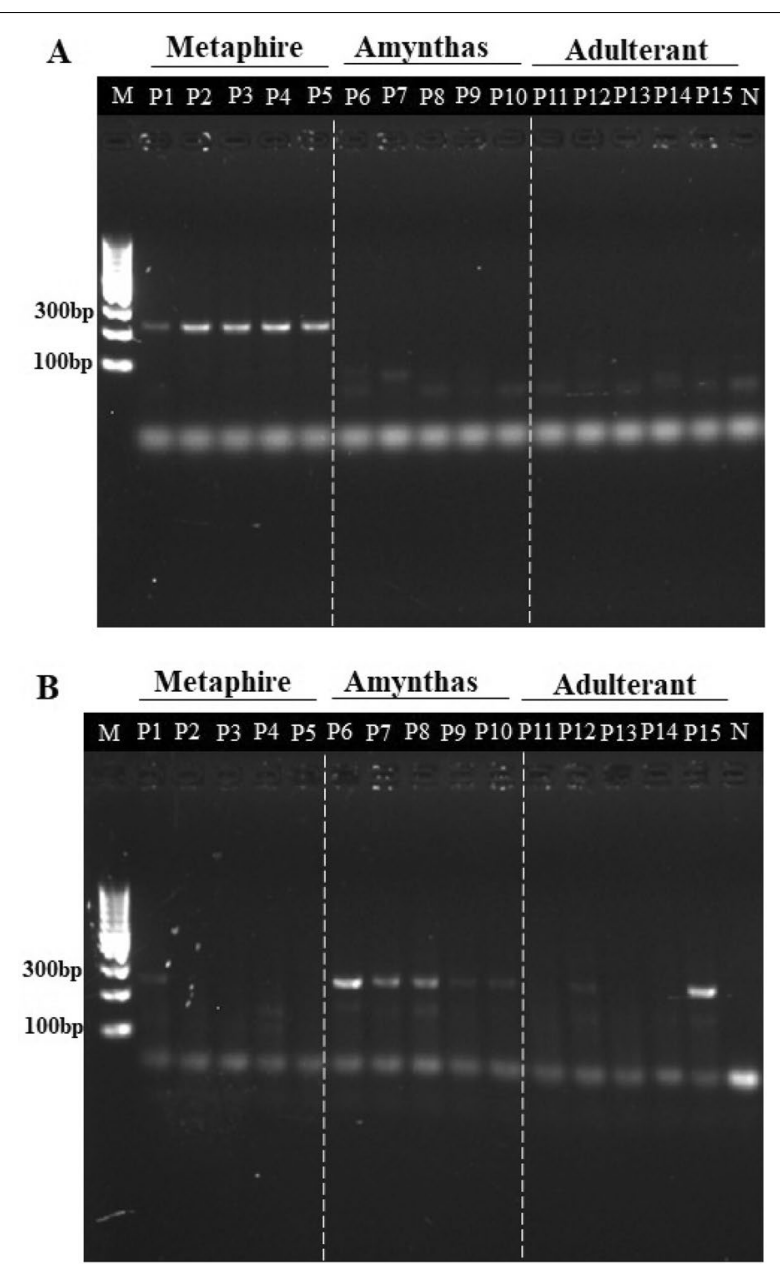

Fig. 1 Specific primers for amplifying DNA extracted from the crude drugs of Pheretima. DNA was amplified using specific primers, (a) primers MF2R2 for Metaphire genus and (b) primers AF3R1 for Amynthas genus, respectively. Adulterant numbered P15 could also be amplified using primers AF3R1, but with sequence and BLAST to the adulterant. PCR without DNA template was used as negative control (N). M:DNA ladders

obtained from samples of Metaphire genus and most of the adulterants. One of the adulterants, sample P15, was also amplified by AF3R1 and found as M. californica by DNA sequencing. It was identified to be M. californica by using the universal primers of COI. To sum up, as for the Pheretima, primers MF2R2 could distinguish M. vulgaris and M. guillelmi from the Amynthas genus and the adulterants. Whereas, though primers AF3R1 could differentiate $A$. aspergillus from the Metaphire genus, sequencing and BLAST need to be done to confirm the amplicon is the genuine species or the adulterant (Additional file 2: Fig. S2-2).

Among all the medicinal materials tested above, P1P4 were provided by Shaanxi Buchang Pharmaceuticals

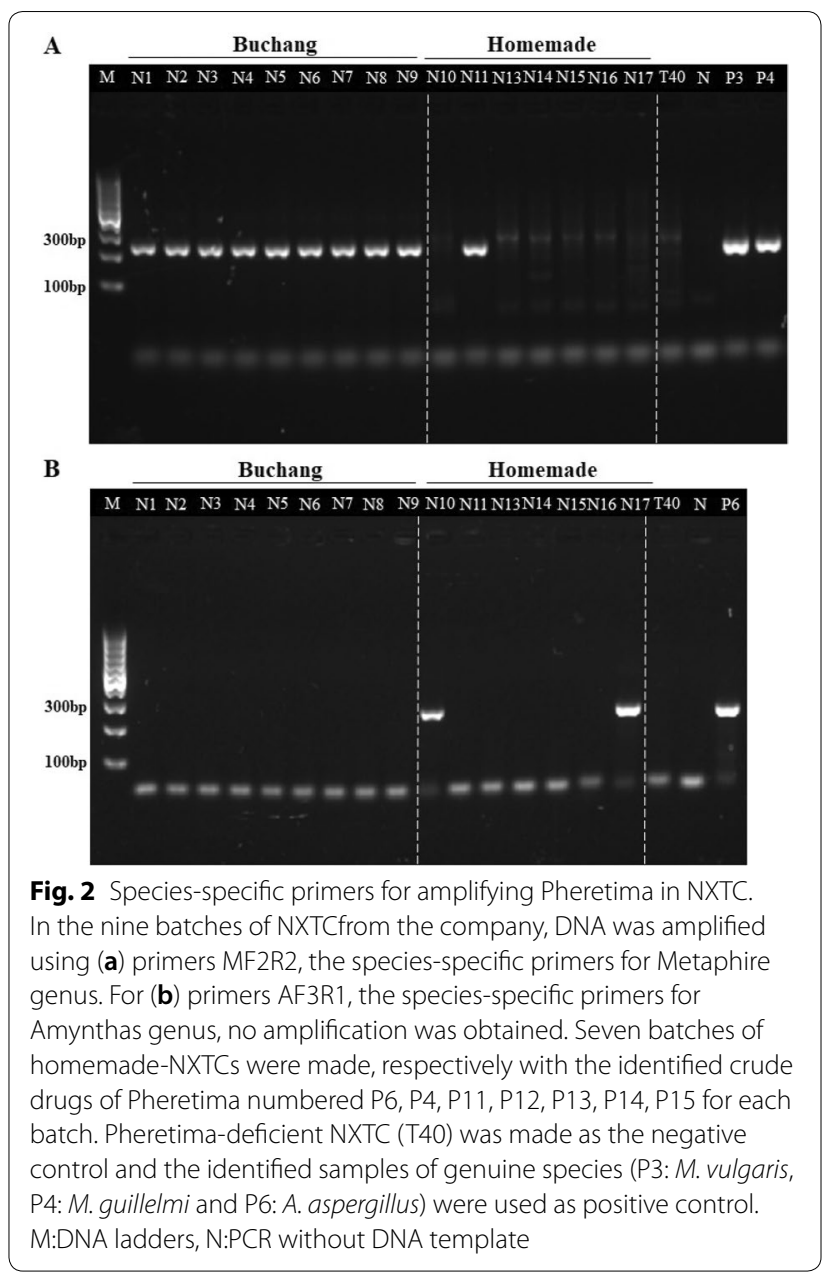

Co., Ltd. (Shaanxi, China) and were identified as $M$. vulgaris or M. guillelmi (Table 1). On this occasion, the results shown above indicated that the primers MF2R2 could be used to identify the genuine species of Pheretima used, which would contribute to the quality control of NXTC at product manufacturing.

\section{Identification of the animal drugs in NXTC by species-specific PCR}

Based on the successful identification of crude drugs, all the specific primers were tested in authenticating the three animal drugs in nine batches of NXTC. It should be noticed that since the company purchased the crude drugs from fixed suppliers, in principle only $M$. vulgaris and M. guillelmi would be detected in NXTC.

As shown in Fig. 2b, no amplification was obtained in NXTC while using primers AF3R1, indicating that there is no A. aspergillus in NXTC. However, 232-bp fragments were amplified in NXTC using primer MF2R2 (Fig. 2a) and all the amplicons were sequenced and BLAST to $M$. vulgaris, which is one of the two species the company 
used (Additional file 3). To find whether primers MF2R2 and AF3R1 could authenticate Pheretima in other NXTC, we tested seven batches of homemade NXTC, in which A. aspergillus, M. guillelmi and the other five batches of adulterants were included respectively. With primers MF2R2, M. guillelmi could be identified when it was included in sample N11 and the NXTC including the adulterants could also be differentiated. At the same time, A. aspergillus and the other adulterants could be distinguished when they were included in NXTC. As revealed above, the Pheretima that the company used include two species, but only $M$. vulgaris was detected in these nine batches of NXTC, which probably due to the use of $M$. vulgaris more frequently. Accordingly, with primer MF2R2, it could be confirmed whether the NXTC to be tested contains the genuine species of Pheretima.

\section{The sensitivity test of NXTC using specific primers for Pheretima}

DNA templates of NXTC (50 ng/ $\mu \mathrm{l})\left(5^{0}\right)$ were fivefold serial-diluted $\left(5^{1}\right.$ to $\left.5^{7}\right)$ and were amplified using specific primers for Pheretima. Every batch of concerned NXTC was tested and repeated once. Results showed that the animal drugs' DNA was detectable with the DNA concentration of NXTC around $50 \mathrm{ng} / \mu \mathrm{l}$ for all the specific primers, and even with lower concentration (Figs. 3 and 4). Taken all the concerned NXTC into consideration,

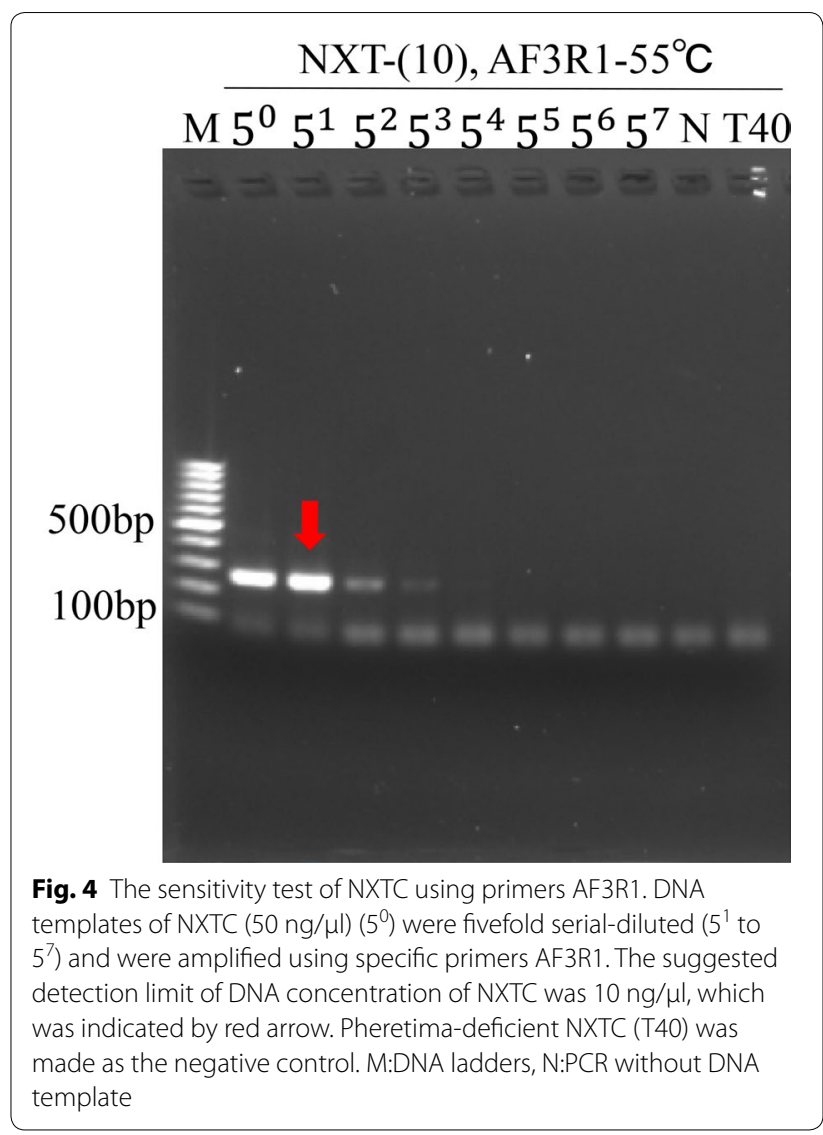

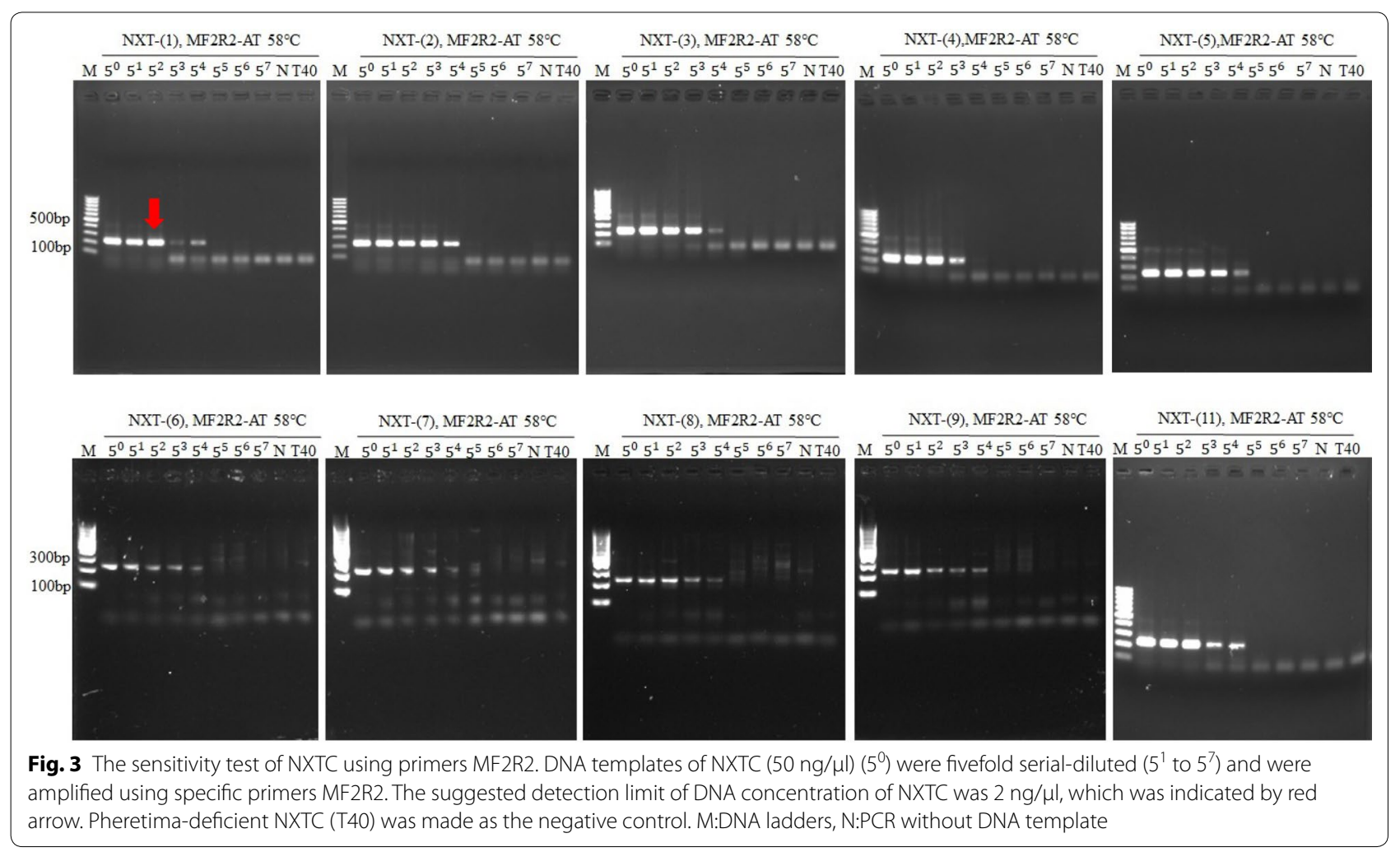


we suggest that the DNA concentration of tested NXTC should be at least $2 \mathrm{ng} / \mu \mathrm{l}$ with primers MF2R2 or $10 \mathrm{ng} /$ $\mu \mathrm{l}$ with primers AF3R1 if this method was applied in the quality control of NXTC.

\section{Discussion}

Accurate species identification is crucial to the authenticity, safety and efficacy of medicinal products. In addition to the conventional methods, such as organoleptic and chemical methods, species identification of animalderived products mainly relies on protein [24] and DNA $[25,26]$ analysis. Protein analysis is usually suitable for raw materials. But for animal-based TCMs, their proteins are generally destroyed due to the high temperature or high salinity during the process. DNA is more reliable and stable than other macromolecules and is found in all tissues. In ChP 2015, "Principles for molecular identification of traditional Chinese materia medica using DNA barcoding" was established to guide the molecular identification of TCM [27]. DNA barcoding is the use of a short, conserved and standardized sequence of the genome to identify species [22]. The application of DNA barcoding for the species identification of global species has become routine. DNA authentication of Pheretima [19-21] has so far aiming at the identification of single crude drug. For example, Chen WM et al. [21] have established a rapid and accurate method to authenticate a genuine species (A. aspergillus) in the crude drug of Pheretima using primers at $12 \mathrm{~S}$ rRNA region.

The present study is the first to use species-specific sites from DNA barcode to identify Pheretima in NXTC, a complex prescription without effective method to control the quality of its animal drugs. In consideration of the DNA degradation during processing and preservation, species-specific primers for Pheretima were designed to target amplicons of small sizes. COI gene was selected for amplification owing to its advantages in providing deeper phylogenetic insights than any other mitochondrial genes [17] and numerous available sequences in GenBank database.

Compared with the identification of single animal drug, one of the difficulties that we encountered with NXTC is the DNA extraction efficiency. Since NXTC contains thirteen plant drugs which account for $83.3 \%$ of the components, the large number of plant secondary metabolites are likely to cause difficulty in DNA extraction. Hence, a kit for plant DNA extraction was used to remove the plant secondary metabolites, and proteinase $\mathrm{K}$ was then added to digest the animal drugs. Results showed that DNA from the animal drugs was detected with the DNA concentration of NXTC at $50 \mathrm{ng} / \mu \mathrm{l}$ of all the specific primers (Figs. 3 and 4).
Admittedly, more approaches with higher resolution, sensitivity, and throughput can be used for the DNA analysis. For instance, Arulandhu et al. [28] have shown that a multi-locus DNA metabarcoding method based on Illumina MiSeq amplicon sequencing can reproducibly identify plant and animal species in highly complex products such as traditional medicines. Besides, Coghlan et al. [29] used high-throughput sequencing to identify species in 15 complex TCMs presented in the form of powders, tablets, and capsules and revealed that some of them contained currently endangered and protected animals' DNA. However, methods that applied to the quality control of TCM generally should be rapid, with good universality and easy to operate. And these technologies are relatively costly and expertise-dependent, which may not suitable for bulk detection of Chinese patent medicine. Agarose electrophoresis of the PCR-ed samples is indeed less sensitive than those approaches, but the result of sensitivity test (Figs. 3 and 4 ) showed that the method we developed is sensitive enough for the identification of Pheretima.

In general, our work has provided an effective and rapid method to authenticate Pheretima in NXTC, which is a progress compared with the current quality control of Pheretima in NXTC by allowing the manufacturer to easily confirm the presence of Pheretima in the product. All testing methods have their own limitations. In this case, our method cannot identify some artificial adulterant samples that do not contain DNA and does not yield information regarding the concentration of active ingredients, which are common problems in many speciesspecific molecular identification methods for traditional Chinese medicine [30]. However, we have already shown that our method can detect the presence of Pheretima in nine different batches of NXTC of the same manufacturer. Suffice to say, if Pheretima DNA cannot be detected in any one batch of NXTC from the same manufacturer in the future, this result should be alarming, and the manufacturer should refer to the retained starting materials for further authentication and tracking. Certainly, more work need to be done with the quality control of NXTC and a combination of DNA barcoding and other methods like chemical analysis is necessary for a comprehensive quality assessment of Pheretima in NXTC.

\section{Conclusion}

As one of the minister drugs of NXTC, Pheretima is of great importance to the efficacy of the formula. However, effective method for the quality control of animal drugs, especially the authentication of Pheretima, in NXTC is lacking. In this study, species-specific primers for the two genera of Pheretima were designed to identify this animal drug with small-size amplicons at around 
$230-250 \mathrm{bp}$, thereby improving the quality control of NXTC. This work has revealed the possibility of using DNA techniques to authenticate animal drug in a complex prescription.

\section{Supplementary information}

Supplementary information accompanies this paper at https://doi. org/10.1186/s13020-019-0264-7.

Additional file 1. GenBank accession numbers of animal species for primer design

Additional file 2. Multiple alignment of the sequences amplified from the crude drugs of Pheretima with specific primers.

Additional file 3. DNA sequences of amplicons and its percentage similarity to NCBI DNA sequences.

\section{Abbreviations}

NXTC: Naoxintong capsule: TCM: traditional Chinese medicine; COI: mitochondrial cytochrome c oxidase subunit I; BLAST: Basic Local Alignment Search Tool; CFDA: China Food and Drug Administration; TLC: thin layer chromatography.

\section{Acknowledgements}

Not applicable.

\section{Authors' contributions}

WS, PS, and XZ designed the study. XZ and HW performed the experiment. $X Z, W S, P S$, and WP analyzed the data and wrote the manuscript. WS, PS, $X Z$ and $H W$ revised the manuscript. All authors read and approved the final manuscript.

\section{Funding}

Financial support was provided by the Scientific and Technological Transformative Project of Sun Yat-Sen University, China (No. 33000-18825004).

\section{Availability of data and materials}

The datasets supporting the conclusions of this article are included within the article and its additional files.

\section{Ethics approval and consent to participate}

Not applicable.

\section{Consent for publication}

Not applicable.

\section{Competing interests}

The authors declare that they have no competing interests.

\section{Author details}

${ }^{1}$ Guangdong Engineering and Technology Research Center for Quality and Efficacy Reevaluation of Post-Market Traditional Chinese Medicine, Guangdong Key Laboratory of Plant Resources, School of Life Sciences, Sun Yat-Sen University, Guangzhou 510275, China. ${ }^{2}$ Li Dak Sum Yip Yio Chin R \& D Centre for Chinese Medicine, The Chinese University of Hong Kong, Shatin, N.T., Hong Kong, China. ${ }^{3}$ Institute of Chinese Medicine, The Chinese University of Hong Kong, Shatin, N.T., Hong Kong, China. ${ }^{4}$ School of Life Sciences, The Chinese University of Hong Kong, Shatin, N.T., Hong Kong, China.

Received: 30 June 2019 Accepted: 23 September 2019 Published online: 30 September 2019

\section{References}

1. Chinese-Pharmacopoeia-Commission. Pharmacopoeia of People's Republic of China, in part 1. Beijing: China Medical Science Press (Chin); 2015. p. 122-3.
2. Group of Chinese Experts Consensus on Clinical Application of Naoxintong Capsule. Experts Consensus on Clinical Application of Naoxintong Capsule. Chin J Integr Med. 2018;24:232-6.

3. Zheng X, Zhang YJ. Clinical efficacy of Buchang Naoxintong Capsules in the treatment of stable angina pectoris (Chin). Chin Med Herald. 2016;13:137-40.

4. Liang QE, Cai YF, Chen RX, Chen WH, Chen LG, Xiao Y. The effect of Naoxintong Capsule in the treatment of patients with cerebral infarction and carotid atherosclerosis: a systematic review and meta-analysis of randomized trials. Evid Based Complement Alternat Med. 2018;2018:5892306.

5. Yang $L, G$ Go Y. Meta-analysis of Naoxintong capsules on transient ischemic attack (Chin). Pharm Clin Chin Meter Medica. 2015;31:202-7.

6. Wang LQ. Naoxintong Capsule treatment of vertebrobasilar insufficiency in 100 cases (Chin). Guangming Tradit Chin Med. 2011;26:87-8.

7. Chen YJ, Zhang P, Luo Y, Liu GX, Jiang FS, Peng Z. Effect of Naoxintong Capsule on carotid atherosclerotic plaque and CRP and Hey in patients with cerebral infarction (Chin). Liaoning J Tradit Chin Med. 2017:44:1920-1.

8. Zhong F, Li W, Li YH. Effect of Naoxintong Capsule on secondary prevention of myocardial infarction (Chin). Chin J Integr Med Cardio Cerebrovasc Dis. 2014;12:416-8

9. Gu GG. Shennong's materia medica classic. Annotated by Yang PJ. Beijing: Academy Press (Chin); 2007. p. 292.

10. Huang CQ, Li W, Zhang QF, Chen WM, Zhang HC, Ni YX. Anti-inflammatory activities of Guang-Pheretima extract in lipopolysaccharide-stimulated RAW 264.7 murine macrophages. BMC Complement Altern Med. 2018;18:46

11. Balamurugan M, Parthasarathi K, Ranganathan LS, Cooper EL. Hypothetical mode of action of earthworm extract with hepatoprotective and antioxidant properties. J Zhejiang Univ Sci B. 2008;9:141-7.

12. Wang F, Wang C, Li M, Zhang JP, Gui LL, An XM, Chang WR. Crystal structure of Earthworm fibrinolytic enzyme component B: a novel, Glycosylated two-chained Trypsin. J Mol Biol. 2005;348:671-85.

13. Chu XP, Xu ZH, Wu DZ, Zhao AH, Zhou MM, Qiu MF, Jia W. In vitro and in vivo evaluation of the anti-asthmatic activities of fractions from Pheretima. J Ethnopharmacol. 2007;111:490-5.

14. Deng ZH, Gao SS, Xiao X, Yin N, Ma SY, Li WP, Li YS. The effect of earthworm extract on mice $\mathrm{S} 180$ tumor growth and apoptosis. Biomed Pharmacother. 2019;115:108979.

15. Fu YT, Chen KY, Chen YS, Yao CH. Earthworm (Pheretima aspergillum) extract stimulates osteoblast activity and inhibits osteoclast differentiation. BMC Complement Altern Med. 2014:14:440.

16. Cheng P, Ye MX, Yan YC, Wang KQ. A survey on the resources of Dilong and identification of the drug available on Chinese market (Chin). Chin Tradit Herbal Drugs. 1997;28:492-5.

17. Ming LI, Cao H, But PH, Shaw PC. Identification of herbal medicinal materials using DNA barcodes. J Syst Evol. 2011;49:271-83.

18. Yang F, Ding F, Chen H, He MQ, Zhu SX, Ma X, Jiang L, Li HF. DNA barcoding for the identification and authentication of animal species in traditional medicine. Evid Based Complement Alternat Med. 2018;2018:1-18.

19. Ma M, Li W, Gong L, Lu RS. DNA barcode identification of Pheretima medicinal material and its adulterants based on COI and 16SrRNA gene sequence (Chin). Tradit Chin Drug Res and Clin Pharmacol. 2014:25:595-8.

20. Tian N, Wei YC, Yuan Y, Jin Y, Yang Q, Zhao YY, Jing C. Identification of Pheretima by multiplex Allele-specific PCR (Chin). Chin J Exp Tradit Med Formul. 2019;25:124-9.

21. Chen WM, Ma M, Gong L, Li W. Specific polymerase chain reaction molecular identification of Pheretima aspergillum (E. Perrier) (Chin). J Guangzhou Univ Tradit Chin Med. 2015;2:499-500.

22. Hebert DN, Cywinska A, Ball SL, DeWaard JR. Biological identifications through DNA barcodes. Proc R Soc Lond B Biol Sci. 2003;270:313-21.

23. Hall TA. BioEdit: a user-friendly biological sequence alignment editor and analysis program for Windows 95/98/NT. Nucleic Acids Symp Ser. 1999:41:95-8.

24. Soares R, Franco C, Pires E, Ventosa M, Palhinhas R, Koci K, de Martinho Almeida A, Varela Coelho A. Mass spectrometry and animal science: protein identification strategies and particularities of farm animal species. J Proteomics. 2012;75:4190-206.

25. Bottero MT, Dalmasso A. Animal species identification in food products: evolution of biomolecular methods. Vet J. 2011;190:34-8. 
26. Rodriguez-Ramirez R, Gonzalez-Cordova AF, Vallejo-Cordoba B. Review: authentication and traceability of foods from animal origin by polymerase chain reaction-based capillary electrophoresis. Anal Chim Acta. 2011;685:120-6.

27. Chinese-Pharmacopoeia-Commission. Pharmacopoeia of People's Republic of China, in part 4. Beijing: China Medical Science Press (Chin); 2015. p. 383-5.

28. Arulandhu AJ, Staats M, Hagelaar R, Voorhuijzen MM, Prins TW, Scholtens I, Costessi A, Duijsings D, Rechenmann F, Gaspar FB, Crespo MTB, HolstJensen A, Birck M, Burns M, Haynes E, Hochegger R, Klingl A, Lundberg L, Natale C, Niekamp H, Perri E, Barbante A, Rosec JP, Seyfarth R, Sovova T, Moorleghem CV, Ruth SV, Peelen T, Kok E. Development and validation of a multi-locus DNA metabarcoding method to identify endangered species in complex samples. GigaScience. 2017;6:1-18.
29. Coghlan ML, Haile J, Houston J, Murray DC, White NE, Moolhuijzen P, Bellgard MI, Bunce M. Deep sequencing of plant and animal DNA contained within traditional chinese medicines reveals legality issues and health safety concerns. PLoS Genet. 2012;8:e1002657.

30. Han JP, Pang XH, Liao BS, Yao H, Song JY, Chen AL. An authenticity survey of herbal medicines from markets in China using DNA barcoding. Sci Rep. 2016;6:18723

\section{Publisher's Note}

Springer Nature remains neutral with regard to jurisdictional claims in published maps and institutional affiliations.
Ready to submit your research? Choose BMC and benefit from:

- fast, convenient online submission

- thorough peer review by experienced researchers in your field

- rapid publication on acceptance

- support for research data, including large and complex data types

- gold Open Access which fosters wider collaboration and increased citations

- maximum visibility for your research: over 100M website views per year

At BMC, research is always in progress.

Learn more biomedcentral.com/submissions 